Supporting information:

\title{
Suppression of Point Defects for Band Edge Engineering in a Semiconducting Photocatalyst
}

\author{
Xin Wang, $\uparrow$ Huiting Huang, $\dagger$ Jiajia Wang,,$\dagger$ Z Zhaosheng Li, $\dagger^{*}$ Zhigang Zou $\dagger$ \\ $\dagger$ National Laboratory of Solid State Microstructures, School of physics, College of Engineering and Applied \\ Sciences, Nanjing University, 22 Hankou Road, Nanjing 210093, China. \\ $\$$ College of Mechanics and Materials, Hohai University, 8 Fochengxi Road, Nanjing 210098, China.
}

\section{Competitive stabilities of hydrogenic impurities in $\mathrm{Ta}_{3} \mathbf{N}_{5}$}

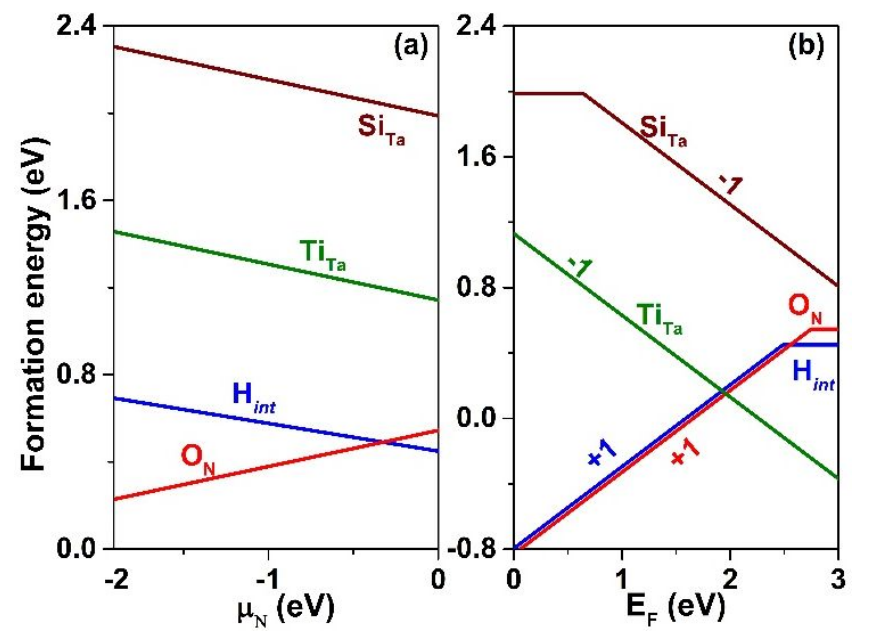

Figure S1. Formation energies of $\mathrm{Ta}_{3} \mathrm{~N}_{5}$ containing $\mathrm{H}_{\text {int }}, \mathrm{O}_{\mathrm{N}} \mathrm{Si}_{\mathrm{Ta}}$ and $\mathrm{Ti}_{\mathrm{Ta}}$ impurities at (a) neutral states without any impurity ionizations and (b) charge states with ionizations.

In a common case, point defects in $\mathrm{Ta}_{3} \mathrm{~N}_{5}$ are described with formation energies of defected $\mathrm{Ta}_{3} \mathrm{~N}_{5}$ supercell at neutral states and charge states. The formation energy at neutral state is defined as a function respecting to the element chemical potentials:

$$
E_{\text {Defect }}^{f}=E_{\text {Defect }}^{t}-E_{\text {Bulk }}^{t}+\sum_{i} n_{i} \Delta \mu_{i}
$$

where $E_{\text {Defect }}^{t}$ is the total energy of a supercell containing the defect, $E_{\text {Bulk }}^{t}$ is the total energy of the pure bulk Ta $\mathrm{N}_{5}$ supercell, $\mathrm{n}_{i}$ is the number of constitute $i(i=\mathrm{H}, \mathrm{N}, \mathrm{O}, \mathrm{Ta}), \Delta \mu_{i}$ is the chemical potential of constitute $i$. Chemical potentials of $\mathrm{H}, \mathrm{N}, \mathrm{O}, \mathrm{Si}$, Ta and Ta are limited by the formation energies of their most stable compounds to avoid the secondary phases, and the detailed procedures are described in the previous works of our group. Here the TaON is taken as an example: to avoid the secondary phase, the following equation should be satisfied:

$$
\Delta \mu_{N}+\Delta \mu_{O}+\Delta \mu_{T a}<E_{T a O N}^{f}
$$

The formation energy at charge state is respecting to the Fermi level:

$$
E_{\text {Defect }}^{f}\left(X^{q}\right)=E_{\text {Defect }}^{t}\left(X^{q}\right)-E_{\text {Bulk }}^{t}+\sum_{i} n_{i} \Delta \mu_{i}+q\left(E_{F}+E_{V}+\Delta V\right)
$$

where $E_{\text {Defect }}^{t}\left(X^{q}\right)$ is the total energy of supercell containing defect $X$ at charge state $q, \mathrm{E}_{\mathrm{F}}$ is the Fermi level referenced to the VBM $\left(\mathrm{E}_{\mathrm{V}}\right)$ of the pure model, $\Delta \mathrm{V}$ is correction term derived from electrostatic potential difference of the $\mathrm{Ta}_{3} \mathrm{~N}_{5}$ 
supercell containing defect $X^{q}$ and the pure $\mathrm{Ta}_{3} \mathrm{~N}_{5}$ supercell.

Formation energies of defected $\mathrm{Ta}_{3} \mathrm{~N}_{5}$ supercells at neutral states are calculated according to Equation $\mathrm{S} 1$ and shown in Figure S2(a). As can be seen, the formation energies of hydrogen, oxygen, silicon and titanium are positive values, demonstrating their limited concentrations in $\mathrm{Ta}_{3} \mathrm{~N}_{5}$. Besides, the formation energies of acceptors silicon $\left(\mathrm{Si}_{\mathrm{Ta}}\right)$ and titanium $\left(\mathrm{Ti}_{\mathrm{Ta}}\right)$ are much higher than the donors hydrogen $\left(\mathrm{H}_{\text {int }}\right)$ and oxygen $\left(\mathrm{O}_{\mathrm{N}}\right)$, demonstrating that the donors are much more easily formed in $\mathrm{Ta}_{3} \mathrm{~N}_{5}$, and accordingly the concentration of donors will be much higher than the acceptors.

Formation energies above indicate the competitive stabilities of the impurities without any ionizations. Based on the defect chemistry, the defects $\mathrm{H}_{i n}, \mathrm{O}_{\mathrm{N}} \mathrm{Si}_{\mathrm{Ta}}$ and $\mathrm{Ti}_{\mathrm{Ta}}$ have weak attractions on their electrons (or holes), and they are easily ionized. Thermodynamics of their ionizations is usually described by charged formation energies, which is written as a function to the Fermi level and calculated according to Equation S3. In this study, the charged formation energies are calculated in the $\mathrm{N}$ rich condition (i.e. $\mu_{\mathrm{N}}=0$ ), catering to the $\mathrm{N}$ rich atmosphere in synthetic process. As shown in Figure S1(b), the formation energies of unionized defects (horizontal lines) have intersections with their charged formation energies (oblique lines). The intersections are called transition levels and they indicate the Fermi levels where the impurities start to ionize into charge states. Based on Figure S2(b), the transition levels of $\mathrm{H}_{\text {int }}$ and $\mathrm{O}_{\mathrm{N}}$ are close to the CBM, indicating two shallow-level donors. Similarly, transition levels of $\mathrm{Si}_{\mathrm{Ta}}$ and $\mathrm{Ti}_{\mathrm{Ta}}$ are close to the VBM, indicating two shallow-level acceptors.

In practice, the extremely high acceptor dopants such as $3 \sim 10 \%$ Ti are applied for $\mathrm{Ta}_{3} \mathrm{~N}_{5}$ doping. It shall be noted that, because the high formation energies of acceptors than donors, heavy acceptor doping on the $\mathrm{Ta}_{3} \mathrm{~N}_{5}$ (which has heavy inherent donors) will be difficult. For one hand, the high formation energies of acceptors indicate that the acceptors are hardly formed in $\mathrm{Ta}_{3} \mathrm{~N}_{5}$ (comparing with the inherent donors); on the other hand, because the oxides and nitrides of the doped elements have lower formation energies, the doped acceptors are easily segregated for oxide/nitride segregations. In fact, because of the limits of phase separations and requirements of lattice stability, if the concentration of donors is high enough (e.g. about 20\%), the acceptor doping cannot compensate all the donors. As a result, the band edge engineering is effectual even though the acceptor feeding is extremely high.

\section{Effective mass approximation for hydrogenic impurities in $\mathrm{Ta}_{3} \mathbf{N}_{5}$}

Because the point defects in this study are hydrogenic impurities whose ionized states have only unit charge differences to its neutral states (i.e., $\mathrm{H}_{\text {int }}, \mathrm{O}_{\mathrm{N}} \mathrm{Si}_{\mathrm{Ta}}$ and $\mathrm{Ti}_{\mathrm{Ta}}$ ), the effective mass approximation is applied to calculate the ionization energies and defect energy levels. The approximation makes use of effective masses, which can be calculated as following equation:

$$
\frac{m^{*}}{m_{e}}=\frac{\mathrm{h}^{2}}{2|a|}
$$

where $\mathrm{m}_{\mathrm{e}}$ is the electron rest mass, $\mathrm{h}$ is reduced planks constant, a is the second derivative of the curve in conduction band (for electron) and valance band (for hole).

To obtain the parameter $\alpha$, band structures of $\mathrm{Ta}_{3} \mathrm{~N}_{5}$ with defects $\mathrm{H}_{\text {int }}, \mathrm{O}_{\mathrm{N}}, \mathrm{Si}_{\mathrm{Ta}}$ and $\mathrm{Ti}_{\mathrm{Ta}}$ are calculated by Densityfunctional theory (DFT) as implemented in the Vienna ab initio simulation package (VASP). Generalized-gradient approximation (GGA) in Perdew-Burke-Ernzerhof (PBE) is applied for structural relaxations and Heyd-ScuseriaErnzerhof (HSE) methods are employed for further calculations on band structures. The parameters for the HSE calculations are $\alpha=0.25$ and $\omega=0.2 \AA^{-1}$, which refer to the HSE06 functional. For the integration in the reciprocal space, a converged energy is reached for a k-point grid fineness of $0.05 \mathrm{eV} \bullet \AA^{-1}$ for $2 \times 2 \times 1$ bulk $\mathrm{Ta}_{3} \mathrm{~N}_{5}$ primitive cell (space group: $\mathrm{Cmcm}$ ). Cut-off energy for geometry optimization is $500 \mathrm{eV}$ and the Brillouin zone integrations are $4 \times 4 \times 4$ k-point meshes, which have been examined enough for computational accuracy. Band structures of the defected $T_{3} \mathrm{~N}_{5}$ are calculated by redundant k-points with zero weight during HSE06 calculations (see Figure S1).

The effective mass approximation makes use of conductivity effective masses in $\mathrm{Ta}_{3} \mathrm{~N}_{5}$, which will be calculated as the harmonic means of effective mass along Bragg vectors: 


$$
\frac{1}{m_{c}^{*}}=\frac{1}{3}\left(\frac{1}{m_{[100]}^{*}}+\frac{1}{m_{[010]}^{*}}+\frac{1}{m_{[001]}^{*}}\right)
$$

where $m_{[\mathrm{ggl}]}^{*}$ is the effective mass along different crystal orientations. Similar to the Equation S4, the conductivity effective mass of electrons are calculated based on the effective masses of electrons, and the conductivity effective mass of holes are based on the effective masses of holes.
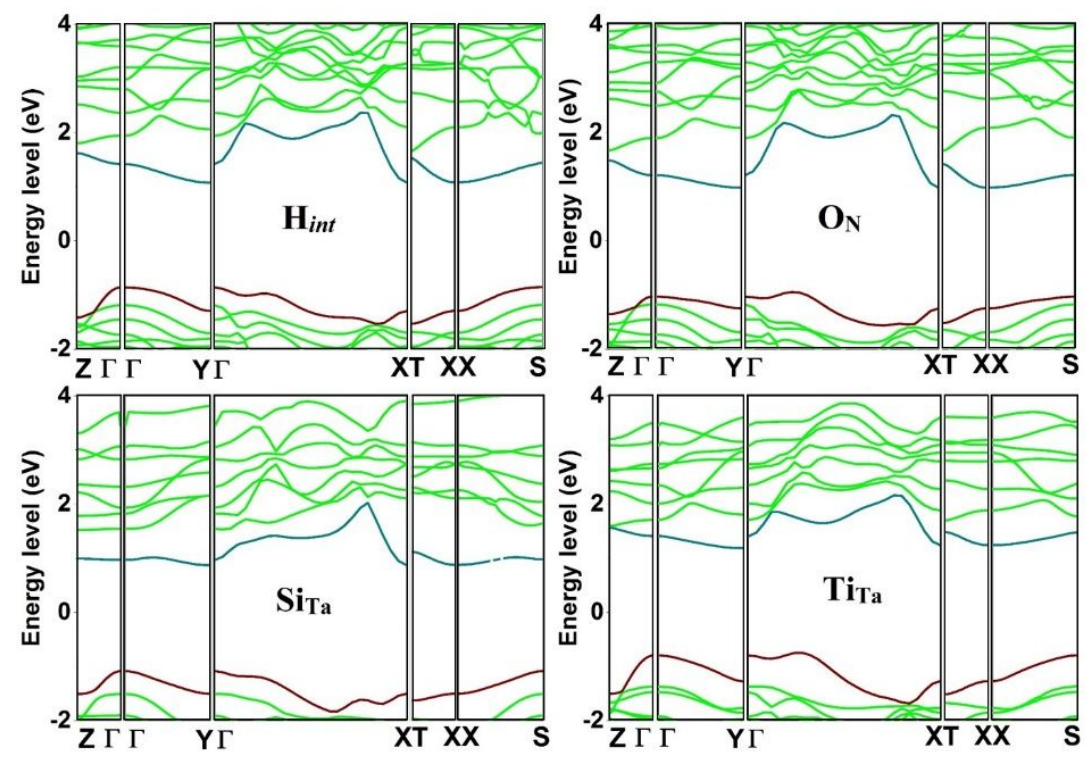

Figure S2. Band structures of $\mathrm{Ta}_{3} \mathrm{~N}_{5}$ containing $\mathrm{H}_{\text {int }}, \mathrm{O}_{\mathrm{N}}, \mathrm{Si}_{\mathrm{Ta}}$ and $\mathrm{Ti}_{\mathrm{Ta}}$ defects. The band structures are obtained by HSE06 method with zero-weight k-points.

\section{Electric properties of $\mathrm{Ta}_{3} \mathrm{~N}_{5}$ with heavy hydrogen and oxygen donors}

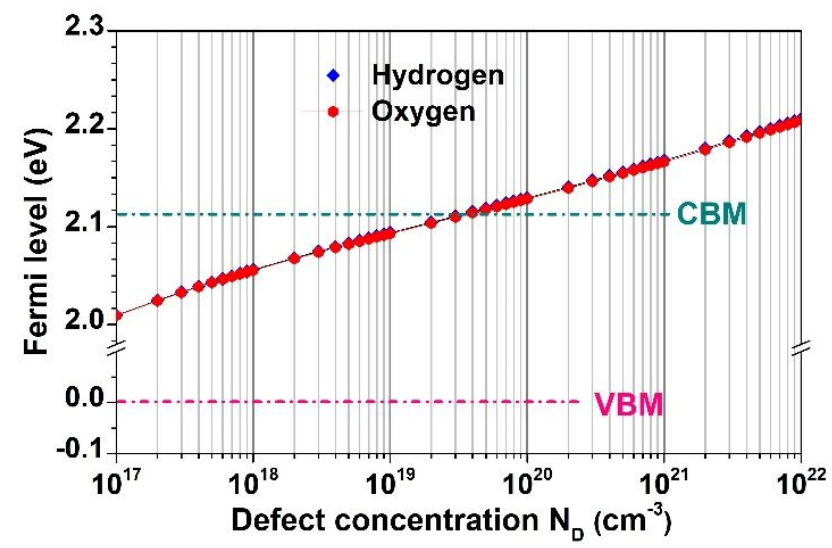

Figure S3. Fermi level of $\mathrm{Ta}_{3} \mathrm{~N}_{5}$ with heavy hydrogen and oxygen donors.

For nondegenerate $\mathrm{Ta}_{3} \mathrm{~N}_{5}$ whose Fermi level is lower than CBM (see Figure S3), the hole concentration is limited by:

$$
n p=2.33 \times 10^{31}\left(\frac{m_{d e}^{*} m_{d h}^{*}}{m_{e}^{2}}\right)^{3 / 2} T^{3} \exp \left(-\frac{E_{g}}{k_{B} T}\right)
$$

where $\mathrm{n}$ and $\mathrm{p}$ are the concentrations of electron and hole carriers, respectively; $\mathrm{T}$ is temperature (at Kelvin), $\mathrm{E}_{\mathrm{g}}$ is band gap of $\mathrm{Ta}_{3} \mathrm{~N}_{5}, m_{d e}^{*}$ and $m_{d h}^{*}$ are the effective masses of conduction bands and valence bands, which are obtained as the geometric means of effective masses along Bragg vectors. For effective mass of conduction bands: 


$$
m_{d e}^{*}=\left(m_{[100]}^{*} m_{[010]}^{*} m_{[001]}^{*}\right)^{1 / 3}
$$

where the $m_{[\mathfrak{g g}]}^{*}$ is the effective mass of electrons along Bragg vectors. The effective mass of valance bands is calculated based on the effective masses of holes.

Based on the effective masses along Bragg vectors, band gap $2.113 \mathrm{eV}$ and temperature $300 \mathrm{~K}$, the product $n p$ of Ta $\mathrm{N}_{5}$ is obtained as $106 \mathrm{~cm}^{-6}$. This value is very small comparing to the other semiconductors, for example, the $n p$ of GaN is $2.5 \times 10^{13} \mathrm{~cm}^{-6}$ at $300 \mathrm{~K}$. As a result, the hole concentration is extremely small in nondegenerate $\mathrm{Ta}_{3} \mathrm{~N}_{5}\left(\mathrm{e} . \mathrm{g} ., 3.4 \times 10^{-17} \mathrm{~cm}^{-3}\right.$ in $3.2 \times 10^{19} \mathrm{~cm}^{-3}$ donor doped $\mathrm{Ta}_{3} \mathrm{~N}_{5}$ ).

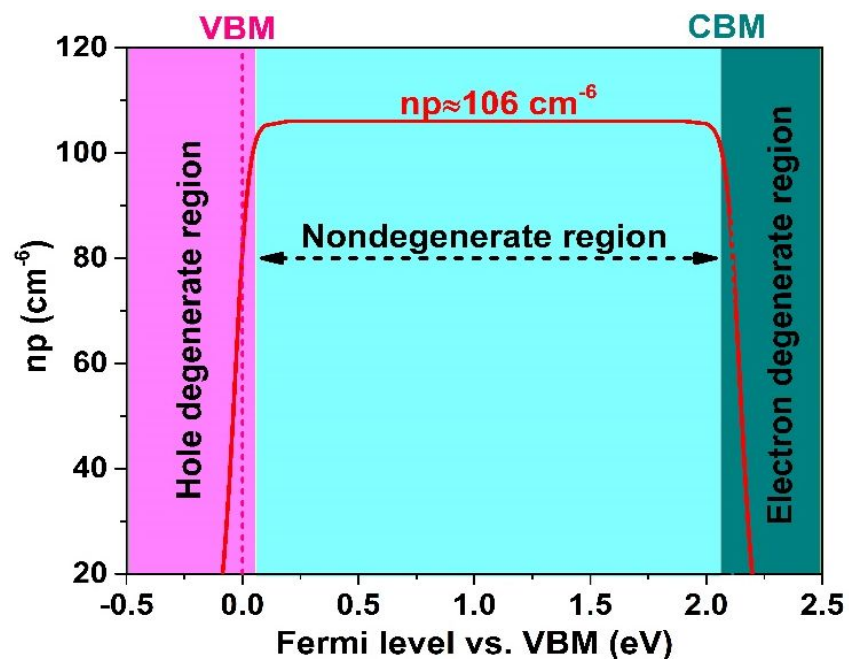

Figure S4. Product of electron and hole concentrations in $\mathrm{Ta}_{3} \mathrm{~N}_{5}$ by Fermi-Dirac statistics.

For $\mathrm{Ta}_{3} \mathrm{~N}_{5}$ samples with heavy hydrogen and oxygen impurities, the Fermi level is higher than the CBM (see Figure S3) and the $\mathrm{Ta}_{3} \mathrm{~N}_{5}$ is degenerate semiconductor. The product of electron and hole concentrations will be lower than $106 \mathrm{~cm}^{-6}$ (see Figure S4), meanwhile the concentration of electron is much higher than its intrinsic concentration (i.e. higher than $10.3 \mathrm{~cm}^{-3}$, see Figure S3). As a result, the concentration of hole carriers will be too small to be ignored.

\section{Several electric parameters for $\operatorname{Ta}_{3} \mathrm{~N}_{5}$}

Despite the charge recombination and semiconductor degeneration, the diffusion coefficient $D_{n, p}$, carrier lifetime $\tau_{n, p}$ and diffusion length $L_{n, p}$ are roughly proportional to the carrier mobility:

$$
\begin{gathered}
D_{n, p}=\mu_{n, p} \frac{k_{B} T}{q} \\
\tau_{n, p}=\mu_{n, p} \frac{m_{n, p}^{*}}{e} \\
L_{n, p}=\sqrt{D_{n, p} \tau_{n, p}}=\mu_{n, p} \sqrt{\frac{m_{n, p}^{*} k_{B} T}{e^{2}}}
\end{gathered}
$$

where $\mu_{n, p}$ is mobility of electrons or holes, $m_{n, p}^{*}$ is the effective mass of electrons or holes, $e$ is electron unit charge, $\mathrm{k}_{\mathrm{B}}$ is Boltzmann constant and $\mathrm{T}$ is the temperature at Kelvin. For a given system with fixed effective masses in room temperature $(\mathrm{T}=300 \mathrm{~K})$, the diffusion coefficient $D_{n, p}$, carrier lifetime $\tau_{n, p}$ and diffusion length $L_{n, p}$ are proportional to the mobility. In this study, these proportional relationships indicate the worse carrier diffusions and shorter lifetimes for carriers of $\mathrm{Ta}_{3} \mathrm{~N}_{5}$ with acceptor doping for band edge engineering, namely worse electric performances of acceptor doped $\mathrm{Ta}_{3} \mathrm{~N}_{5}$ than the $\mathrm{Ta}_{3} \mathrm{~N}_{5}$ with heavy donors. 